\title{
Effect of controlled release salbutamol on nocturnal cough in asthma
}

\author{
E W Hoskyns, A Thomson, E Decker, A Hutchins, H Simpson
}

\begin{abstract}
Sixteen asthmatic children completed a double blind placebo controlled crossover study of controlled release salbutamol (CRS) to assess its efficacy in controlling night time cough. Children with asthma were enrolled into the study. on the basis of a history of persistent cough confirmed by two overnight tape recordings at home. Outcome was measured by two overnight tapes on each medication. Other treatment was unaffected. There was no significant fall in cough counts on CRS. Median scores were 14.5 and 12.0 coughing episodes per night for CRS and placebo respectively. Mean overnight oxygen saturation was identical in both treatment periods but morning peak flow showed a trend towards improvement on CRS. Treatment with CRS does not have a significant effect in control of night cough although it may improve objective measurements of lung function.
\end{abstract}

Cough, particularly at night, is a major symptom of asthma. Although wheeze is a hallmark of asthma, cough may be the more prominent symptom. The relationship of cough and wheeze in asthma and the underlying mechanisms involved have not been fully elucidated. ${ }^{1}$ Night cough in asthma may reflect diurnal variation in peak flow $^{2}$ but the timing of coughing episodes ${ }^{3}$ does not necessarily coincide with that of minimum peak flow. Another possible explanation for increased symptoms at night is that daytime medication with short acting inhaled bronchodilators is insufficient to control night time symptoms.

Oral controlled release salbutamol has been marketed as having a place in control of night time asthma. Twice daily dosage of 4 or $8 \mathrm{mg}$ tablets provides a smooth plasma profile ${ }^{4}$ and comparison of controlled release salbutamol (CRS) with oral theophyllines has shown the preparations to be equally effective in adult asthma. ${ }^{5}$ Preliminary results in children gave similar results. ${ }^{7}$ These studies have also shown that CRS is well tolerated with fewer adverse effects.

The aim of this study was to ascertain the effectiveness of CRS in asthmatic children with persistent night cough.

\section{Subjects}

Twenty seven children aged 6-14 years with moderate to severe asthma who attended the children's chest clinic at Leicester Royal
Infirmary on a regular basis were considered initially for enrolment into the study. The diagnosis of asthma was made on the basis of clincal findings and the demonstration of reversible airway obstruction ( $>15 \%$ increase in peak flow after inhalation of salbutamol). The children were considered for the study if, in addition, there was a history of persistent night cough (of several months' duration) not controlled by current medication or associated with a recent upper respiratory infection. Although cough is a common feature of asthma, persistent cough was uncommon in patients attending the clinic and recruitment took a period of two years. Children already receiving theophylline preparations, long acting $\beta_{2}$ agonists, or oral steroids were excluded, as were those with concurrent chronic systemic disorders.

\section{Methods}

EQUIPMENT

The system for recording nocturnal cough has been described previously. ${ }^{3}$ Essentially it consisted of a directional microphone placed near the child's bed connected to a UHER 4200 tape recorder with a voice activated switch. An acoustic delay ensured that the activating noise was recorded at operating speed by the tape recorder. Ten seconds after cessation of coughing the tape recorder stopped and the time of the episode was recorded on a second channel. The switch threshold was adjusted individually for each child before monitoring. The tape was analysed aurally to exclude extraneous noises and by a specially designed computer program to give the number of 10 second coughing episodes. As the cough counts have a skewed distribution, statistical comparisons were made using $\log _{\mathrm{e}}$ cough counts.

Overnight arterial oxygen saturation $\left(\mathrm{SaO}_{2}\right)$ and pulse rate (averaged over three seconds) were recorded at one minute intervals using a Biox 3700 pulse oximeter attached to a Squirrel data logger (SQ32-3V/IL/3D, Grant Instruments Cambridge Ltd). The finger probe was firmly attached to the child with tape. The overnight data were then edited to exclude any episodes of poor probe connection and the mean overnight saturation calculated.

Each child was given a Wright mini peak flow meter to use at home. Other lung function tests were performed in the respiratory laboratory. Lung volumes and airway resistance were measured in a whole body plethysmograph (Jaeger Ltd) and expiratory flow rates by an electronic spirometer (Gould). Measurements were made 
before and after inhalation of bronchodilator and all results were converted to percentage predicted for height using standard published data.

\section{TRIAL DESIGN}

The trial consisted of one week run-in period followed by a double blind crossover with one week each of CRS $4 \mathrm{mg}$ (Volmax, Glaxo UK) or placebo twice daily. During the run-in period placebo tablets were taken and the child asked to complete a standard diary card (with grades 0-3 for severity of symptoms of wheeze, day and night cough, exercise tolerance, and nasal symptoms) and twice daily peak flow recordings (7-8 am and 7-10 pm) using the Wright mini peak flow meter. During the week the child attended the lung function laboratory for assessment of lung function. On days 6 and 7 of the run-in week, the family were visited at home and overnight recordings of cough and $\mathrm{SaO}_{2}$ were made. At the same time the diary card was checked for completeness and inquiries made about compliance with medication and ability to swallow the tablets whole. If the child had completed the diary card, had taken the placebo satisfactorily, and had recorded at least 20 coughing episodes over the two nights, he/she was entered into the crossover phase.

Diary card requirements, peak flow, and overnight monitoring (cough, $\mathrm{SaO}_{2}$, and heart rate) in the second and third weeks were the same as for the run-in week; during this period each child was randomised to receive either CRS or placebo first. Deterioration in the control of asthma resulting in the prescription of oral steroids or admission to hospital was regarded as a treatment failure and the child withdrawn from the study. The Leicester Health Authority ethical committee approved the study and informed consent was given by parents before enrolment.

\section{SAMPLE SIZE}

The main outcome indicator of the trial was change in night cough. As the number of coughing episodes was not normally distributed it was expressed as $\log _{e}$ cough. Using the $S D$ of $\log _{e}$ cough values from previous studies in similar children of 0.72 (unpublished observations), nine children would be needed to show a $50 \%$ reduction and 15 children to show a $45 \%$ reduction in number of coughing episodes, assuming a power for the study of $80 \%$.

\section{Results}

Sixteen of the original 27 children completed the study. The 11 failures were excluded at the end of the run-in period. Six children did not meet the entry criteria of $>20$ coughing episodes over two nights, and five did not fill in the diary cards satisfactorily. None was withdrawn on medical grounds.

Table 1 gives details of the children who completed the study. All were being treated with short acting inhaled $\beta_{2}$ agonists and 13 were also receiving inhaled steroid medication. Lung function tests reflect the range of daytime
Table 1 Details of children studied. Results are mean (range) except where stated otherwise

\begin{tabular}{lc}
\hline Age (years) & $9 \cdot 3(4 \cdot 8-14 \cdot 0)$ \\
No of boys & 10 \\
No on medication & 16 \\
Inhaled bronchodilator & 13 \\
Inhaled steroids & \\
No with atopic symptoms & 10 \\
Eczema & 9 \\
Rhinitis & 4 \\
Conjunctivitis & 4 \\
Urticaria & \\
Diary card scores & $1 \cdot 3(0-2 \cdot 5)$ \\
Night cough & $0 \cdot 75(0-3 \cdot 0)$ \\
Night wheeze & $0 \cdot 66(0-2 \cdot 0)$ \\
Nasal symptoms & $0 \cdot 92(0-2 \cdot 7)$ \\
Daytime symptoms & \\
Lung function & $92(26-125)$ \\
Forced expiratory volume (FEV & \\
Forced vital capacity (FVC) & $99(57-120)$ \\
FEV:FVC & $77(38-98)$ \\
Peak flow & $92(23-141)$ \\
Airway resistance & $204(82-748)$ \\
RV:TLC & $35(18-72)$ \\
\hline
\end{tabular}

$\mathrm{FEV}_{0.75}, \mathrm{FVC}, \mathrm{FEV}: \mathrm{FVC}$, peak flow, and airway resistance are expressed as \% predicted for height and residual volume total lung capacity (RV:TLC) as a ratio for which the normal range is approximately $22 \% \pm 8$.

severity in children attending the outpatient chest clinic.

\section{RUN-IN PERIOD}

Mean peak flow during the run-in week was $95 \cdot 9 \%$ (evening) and $89 \cdot 2 \%$ (morning) with mean overnight variability (percentage change from evening to morning) $7 \cdot 1 \%$. Symptom scores (median per day on a scale of $0-3$ ) during the run-in week were: nocturnal cough 1.5 , wheeze 0.75 , limitation of activity 1.75 and nasal symptoms $0 \cdot 25$. On overnight monitoring the median number of coughing episodes per night was 25.5 (range 13-195) and mean $\mathrm{SaO}_{2}$ $95 \cdot 1 \%$.

\section{CROSSOVER PERIOD}

Seven children (44\%) received CRS first and there was no significant order effect for any of the variables measured. There was no significant improvement in $\log _{e}$ cough with CRS (figure). In absolute numbers there was an increase in median cough counts on CRS from $12 \cdot 0$ to $14 \cdot 5$. The $\log _{e}$ cough scores were almost identical (table 2), with confidence intervals for the difference equivalent to $-2 \cdot 0$ to $+1 \cdot 9$ coughing episodes per night. The diary card cough score showed a deterioration of night cough on CRS $(>0.05)$. There was also a deterioration of nasal symptoms on CRS. $\mathrm{SaO}_{2}$ saturation was not affected (mean $=95.8 \%$ on both CRS and placebo). Mean peak flow (evening and morning) improved during the treatment week, the increase in morning peak flow on CRS being greatest $(p=0 \cdot 07)$. Mean peak flow variability overnight on CRS was not significantly reduced. The overnight pulse rate was significantly raised on CRS (mean $=88$ beats/minute compared with 76 beats/minute on placebo, $\mathrm{p}<0.003$ ). On examining the individual paired results, all the children had a higher pulse rate while on CRS.

$\log _{e}$ cough correlated with parental scoring of night cough $(r=0.588, p<0.02)$ during the CRS week but not on placebo. Likewise, there 
Table 2 Effect of treatment on outcome variables

\begin{tabular}{|c|c|c|c|}
\hline & $\begin{array}{l}\text { Mean } \\
\text { CRS }\end{array}$ & $\begin{array}{l}\text { Mean } \\
\text { placebo }\end{array}$ & $\begin{array}{l}p \text { Value } \\
\text { (SE difference) }\end{array}$ \\
\hline $\begin{array}{l}\text { Coughing episodes (median) } \\
\text { Log }_{e} \text { cough }\end{array}$ & $\begin{array}{l}14 \cdot 5 \\
2 \cdot 468\end{array}$ & $\begin{array}{l}12 \cdot 0 \\
2 \cdot 464\end{array}$ & $\begin{array}{l}0.99 \\
0.82\end{array}$ \\
\hline $\begin{array}{l}\text { Diary card scores } \\
\text { Night cough } \\
\text { Night wheeze } \\
\text { Nasal symptoms } \\
\text { Mean night } \mathrm{SaO}_{2}(\%) \\
\mathrm{PF} \text { evening as } \% \text { of predicted value } \\
\mathrm{PF} \text { morning as } \% \text { of predicted value } \\
\mathrm{PF} \text { variability as } \%\end{array}$ & $\begin{array}{c}1 \cdot 2 \\
0 \cdot 84 \\
0 \cdot 94 \\
95 \cdot 8 \\
103 \\
102 \\
1\end{array}$ & $\begin{array}{c}0 \cdot 72 \\
0 \cdot 63 \\
0 \cdot 59 \\
95 \cdot 8 \\
100 \\
94 \\
5\end{array}$ & $\begin{array}{l}0.05 \\
0.48 \\
0.09 \\
0.82(0.3) \\
0.38(3.6) \\
0.07(3.8) \\
0.21(2.9)\end{array}$ \\
\hline
\end{tabular}

Coughing episodes and diary card scores were compared using the Wilcoxon signed rank test, and $\log _{\mathrm{e}}$ cough, mean night SaO ${ }_{2}$, and PF measurements using a Student's paired $t$ test. PF, peak flow.

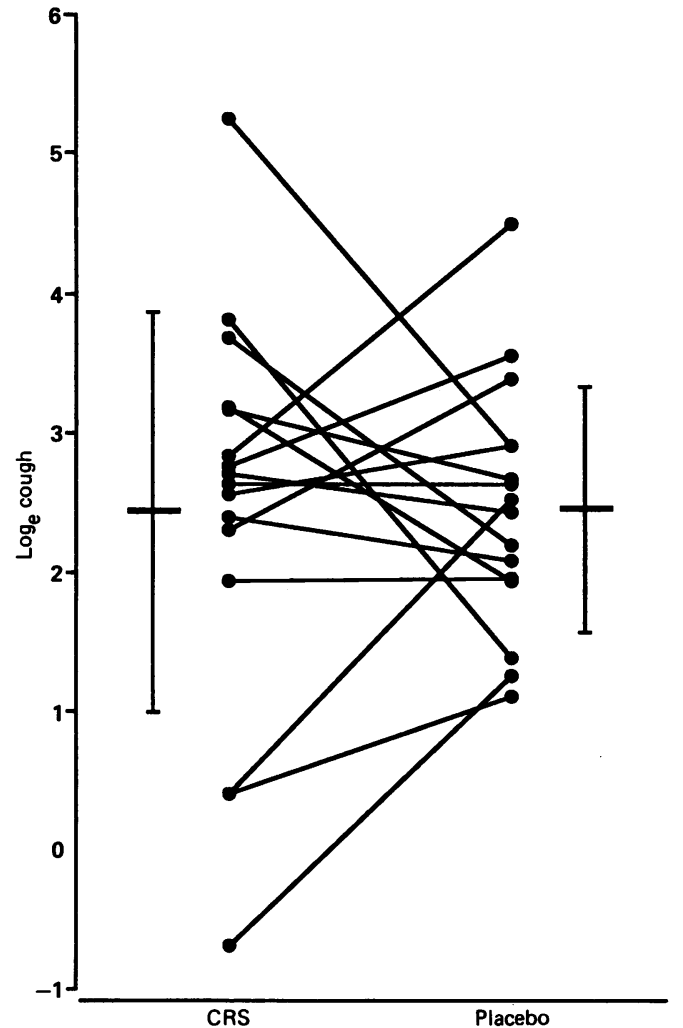

Comparison of $\log _{e}$ cough on controlled release salbutamo $(C R S)$ and placebo. The vertical bars indicate mean (SD).

was a correlation between $\log _{e}$ cough and peak flow $(r=-0.522, p<0.04$ morning and $r=0.726$ $p=0.001$ evening) but only during the treatment week. There was no correlation between $\log _{e}$ cough and either peak flow variability $(r=0 \cdot 18)$ or mean $\mathrm{SaO}_{2}(\mathrm{r}=0 \cdot 29)$.

\section{Discussion}

In asthmatic children with nocturnal cough as a major symptom uncontrolled by inhaled medication, CRS has no significant effect. The broader than expected range of coughing episodes, particularly in the treatment group (SD for $\log _{e}$ cough $=1 \cdot 44$ ), reduces the power of the study but the lack of any trend towards improvement makes it unlikely that larger numbers would have shown a different outcome. The uniform increase in pulse rate, the trend to improvement in morning peak flow, and the good diary card documentation suggest that compliance with medication was satisfactory.

No previous studies have investigated the effect of CRS on night cough. However, other studies have shown that there is no difference between CRS and oral theophylline in terms of peak flow, lung function tests, and symptom scores measured during the daytime. ${ }^{5} 6$ These studies were performed on adult patients with asthma or chronic bronchitis and neither included a placebo group. An unreviewed report of a similar trial in children showed no advantage of CRS over theophylline except that there was a small improvement in the morning and evening peak flow. ${ }^{7}$ Adverse effects were more common with theophylline. In our study there were no reported adverse events or withdrawals related to medication.

One further unreviewed report in children showed a significant improvement in morning and evening peak flow $(16.3 \%$ and $21.5 \%$ respectively) on CRS but was not placebo controlled. ${ }^{8}$ Our study showed mean morning and evening peak flow to be higher on CRS. The difference approached significance for morning values. The study was not designed to show change in peak flow and, with wide SDs, larger numbers would be needed to confirm a significant difference. Improvement in morning peak flow but not in night cough suggests that bronchoconstriction and cough are not directly related in this situation.

Diary card scores of night cough correlated with cough counts but, as shown previously, ${ }^{9}$ these are a poor guide in individual cases. The deterioration in reported cough on CRS is not easy to explain. The association of $\log _{e}$ cough with peak flow in the treatment group but not in the placebo group is probably related to the wider variation in coughing frequency in the former group, making a weak correlation more apparent.

This group of children was chosen because cough was a predominant symptom of their asthma. The severity of asthma varied as measured by peak flow and other lung function tests during the day. The severity of asthma did not correlate with the number of coughing episodes at night for any of these parameters. CRS in the form of Volmax is not an effective treatment of night cough in asthma, even when it results in improvement in peak flow. This supports previous evidence that cough and 
airway narrowing are to some extent independent manifestations of asthma in children with both symptoms.

We are grateful to Allen and Hanbury Ltd for providing Volmax tablets and placebo and for funding of ED and $\mathrm{AH}$.

1 Anonymous. Cough and wheeze in asthma: are they interdependent? Lancet 1988;i:447-8.

2 Soutar CA, Carruthers M, Pickering CAC. Nocturnal asthma and urinary adrenaline and noradrenaline excretion. Thorax and urinary adre

3 Thomson AH, Pratt C, Simpson H. Nocturnal cough in asthma. Arch Dis Child 1987;62:1001-4.

4 Lipworth BJ, Clark RA, Dhillon DP, et al. Single dose and steady-state pharmacokinetics of $4 \mathrm{mg}$ and $8 \mathrm{mg}$ oral salbutamol controlled-release in patients with bronchial asthma. Eur $\mathcal{Y}$ Clin Pharmacol 1989;37:49-52.

5 Higgenbottam TW, Ali Khan MA, Williams DO, et al. Controlled release salbutamol tablets versus aminophylline in the control of reversible airways obstruction. $\mathcal{F}$ Int Med Res 1989;17:435-41.

6 Vyse T, Cochrane GM. Controlled release salbutamol tablets versus sustained release theophylline tablets in the control of reversible obstructive airways disease. 7 Int Med Res 1989;17:93-8.

7 Zeitlin S, Rolles C, Antolainen I, et al. An open, multicentre, cross-over comparison of albuterol controlled release tablets and individually titrated slow release theophylline in the treatment of childhood asthma. Am Rev Resp Dis 1988;137: 33.

8 Weller P. A comparison of a new osmotic pressure mediated oral formulation of salbutamol controlled release tablets (Volmax) and standard salbutamol tablets in the treatment (Volmax) and standard salbutamol tablets in the treatment of childhood asthma

9 Archer LNJ, Simpson H. Night cough counts and diary card scores in asthma. Arch Dis Child 1985;60:473-4.

Now for chickenpox

Chickenpox is the last of the great traditional childhood infections to remain untrammelled by vaccination. Although usually fairly benign it can and does cause much trouble, often disrupting paediatric medical and surgical wards because of its infectivity. It is a cause of severe embryopathy, encephalitis presenting particularly as acute ataxia, and severe disease in the very young and the immunocompromised. We could well manage without it. In the last five years live varicella vaccines have been licensed in Japan, Korea, and several European countries. Now a similar vaccine produced by Merck Sharpe and Dohme has been tested on over 3000 children in America (C Jo White and colleagues, Pediatrics 1991;87:604-10).

Adverse reactions were relatively few. One child developed a fever and a swollen knee 16 days after the vaccination. Mild problems at the injection site, usually pain in the first three days, were experienced by $13 \%$ of children. Four percent developed a rash confined to the injection site and a further $4 \%$ had a varicelliform rash, which was usually mild, occurring at about 14 to 25 days after injection. Viral culture was attempted in 32 cases of post vaccination varicelliform rash and varicella zoster virus was isolated from 11. Nine of these isolates were typed by restriction endonuclease analysis and eight were shown to be wild virus and not the vaccine strain. Which just goes to show, you can't assume nut'n. Less than $1 \%$ of vaccinees had a fever of more than $38.9^{\circ} \mathrm{C}$ within three days and the incidence of fever in the six weeks after vaccination was no greater than might be expected in normal children.

Ninety six percent of vaccinees showed seroconversion at six weeks and all the non-responders given a second dose showed seroconversion after that. Antibodies persisted at one year in almost all cases. The vaccine showed an $86 \%$ efficacy in preventing chickenpox in household contacts in the first year after vaccination and when the disease occurred it was usually mild, the mean number of lesions being 32 compared with about 500 in natural chickenpox. Two mild cases of shingles have been reported in earlier trials of varicella vaccine but the incidence of shingles after vaccination seems to be no higher than after natural infection. Data from previous trials indicate that humoral immunity persists for at least four years. It will be necessary to continue to collect data on the persistence of immunity and the possible need for revaccination but the prospects for control of disease due to varicella zoster virus look good.

Next on the list must surely be respiratory syncytial virus. And then what shall we do in the winter time? Now Jonah, be quiet. What do you mean 'When we got rid of smallpox we got AIDS. When we get rid of chickenpox . . .?' Now that's no way to think, is it? 